\title{
Correlating the Histopathological Features with the Underlying Pathogenesis could Aid in Differentiating Oral Lichen Planus from Related Lesions
}

\author{
K Lizbeth Raju ${ }^{1}$, Shankargouda Patil ${ }^{2}$, Chandini Rajkumar ${ }^{3}$, A Thirumal Raj ${ }^{4}$
}

World Journal of Dentistry (2019): 10.5005/jp-journals-10015-1660

Oral lichen planus (OLP) is considered as a chronic immunemediated inflammatory condition of the oral mucosa. It has a variety of clinical presentations including reticular, erosive, papular, plaquelike, and ulcerative patterns. The major histopathological features include a subepithelial band of inflammatory infiltrates and basal cell degeneration. Examination of the subepithelial inflammation has revealed the predominance of CD8+ lymphocytes. ${ }^{1}$ The recruitment of CD8+ cells occurs as a response to the antigenic stimuli of the oral epithelial keratinocytes. The cytotoxic activity of the CD8+ cells induces apoptosis of the antigenic keratinocytes. ${ }^{2}$ Keratinocytes are responsible for maintaining the basement membrane and vice versa. ${ }^{3,4}$ Thus, apoptotic loss of keratinocytes results in loss of basement membrane integrity which in turn triggers further keratinocyte apoptosis. In addition, the loss of basement membrane integrity allows the migration of a greater number of CD8+ cells triggering large-scale keratinocyte degeneration. With each CD8+ cell attempting to breach through the basement membrane to reach its target antigen, there is an added accumulation of the CD8+ cell in the subepithelial stroma, eliciting the classic appearance of the subepithelial inflammatory band with varying degrees of keratinocyte degeneration.

Although histopathological examination reveals only the above-mentioned characteristic features, a number of other inflammatory mediators have also been identified in OLP using various diagnostic modalities including immunohistochemistry. ${ }^{5}$ These mediators are attributed to be the result of the wide variety of chemokines released by the apoptotic keratinocytes and the CD8+ cells. The chemokines attract additional inflammatory components that sustain the lesion, rendering a chronic course. Cases presenting with these additional inflammatory components are often misdiagnosed as oral lichenoid lesions (OLLs) and oral epithelial dysplasia with secondary lichenoid components. The latter is also referred to as oral lichenoid dysplasia (OLD) as the epithelial dysplasia is the primary pathology and the inflammation is just a reactionary component. Both OLL and OLD have shown to exhibit clinicopathological features similar to those of OLP. ${ }^{6-11}$ In OLL, the antigenic stimuli are from an external factor, which could range from a restorative material to a wide range of medications. In cases of OLD, as mentioned earlier, the inflammatory components are a response to epithelial dysplasia which in turn is often due to a known risk factor such as tobacco/alcohol. ${ }^{6-11}$

Unlike OLL and OLD, details of the triggering/risk factor cannot be elicited through history for OLP. Further, OLP cases could potentially have a coincidental history of oral restorations, a recent change in medications, or a known carcinogenic risk factor such as
${ }^{1}$ Private Dental Clinic, Bengaluru, India

${ }^{2}$ Department of Maxillofacial Surgery and Diagnostic Sciences, College of Dentistry, Jazan University, Jazan, Kingdom of Saudi Arabia

${ }^{3}$ Department of Oral Pathology and Microbiology, Sathyabama University Dental College and Hospital, Chennai, Tamil Nadu, India

${ }^{4}$ Department of Oral Pathology and Microbiology, Sri Venkateswara Dental College and Hospital, Chennai, Tamil Nadu, India

Corresponding Author: A Thirumal Raj, Department of Oral Pathology and Microbiology, Sri Venkateswara Dental College and Hospital, Chennai, Tamil Nadu, India, Phone: +91 8122627810, e-mail: thirumalraj666@gmail.com

How to cite this article: Raju KL, Patil S, Rajkumar C, et al. Correlating the Histopathological Features with the Underlying Pathogenesis could Aid in Differentiating Oral Lichen Planus from Related Lesions. World J Dent 2019;10(4):251-252.

Source of support: Nil

Conflict of interest: None

tobacco/alcohol. ${ }^{7,8}$ In such cases, if the patch test for OLL is negative and if the cessation of the associated factor does not resolve the lesion, then eliciting the self-antigen in the keratinocyte would be an objective confirmation of the disease nature. To conclude, correlating the histopathological features with the underlying pathogenesis (autoimmune response) could aid in understanding the natural history of OLP and in differentiating them from related lesions (OLL and OLD).

\section{References}

1. Sugerman PB, Satterwhite K, Bigby M. Autocytotoxic T-cell clones in lichen planus. Br J Dermatol 2000;142(3):449-456. DOI: 10.1046/j.13652133.2000.03355.x.

2. Sugerman PB, Savage NW, Walsh LJ, et al. The pathogenesis of oral lichen planus. Crit Rev Oral Biol Med 2002;13(4):350-365.

3. Zhou XJ, Sugerman PB, Savage NW, et al. Matrix metalloproteinases and their inhibitors in oral lichen planus. J Cutan Pathol 2001;28(2): 72-82. DOI: 10.1034/j.1600-0560.2001.280203.x.

4. Pullan S, Wilson J, Metcalfe A, et al. Requirement of basement membrane for the suppression of programmed cell death in mammary epithelium. J Cell Sci 1996;109(Pt 3):631-642.

5. Yamamoto T, Nakane T, Osaki T. The mechanism of mononuclear cell infiltration in oral lichen planus: the role of cytokines released from keratinocytes. J Clin Immunol 2000;20(4):294-305. DOI: 10.1023/a:1006671804110.

6. Thirumal Raj A. Oral lichenoid dysplasia and exophytic oral verrucous hyperplasia: mythical entities of oral pathology. Oral Oncol 2017;70:75. DOI: 10.1016/j.oraloncology.2017.05.013. 
7. Thirumal Raj A. Formulating a clinicopathological algorithm for oral lichenoid dysplasia and related lesions. Oral Oncol 2017;71:186-187. DOI: 10.1016/j.oraloncology.2017.06.017.

8. Raj AT, Patil S. Diagnostic flaws in oral lichen planus and related lesions. Oral Oncol 2017;74:190-191. DOI: 10.1016/j.oraloncology.2017.10.003.

9. Patil S, Rao RS, Sanketh DS, et al. Lichenoid dysplasia revisitedevidence from a review of Indian archives. J Oral Pathol Med 2015;44(7):507-514. DOI: 10.1111/jop.12258.
10. Raj AT, Patil S, Gupta A, et al. Reticular whitish gray lesions of the oral mucosa with no habit history: a critical appraisal. World J Dent 2018;9(3):169.

11. Raj AT, Patil S, Gupta AA. True nature of mild epithelial dysplasia in oral lichenoid mucositis - is it a reactionary change to the subepithelial inflammation or represents true malignant potential? A hypothesis. Med Hypotheses 2018;120:48. DOI: 10.1016/j.mehy.2018. 08.011. 